\section{Informationen zur Facharztprüfung Augenheilkunde und Optometrie}

Unserem Antrag für das Jahr 2005 zwei Prüfungstermine zu bewilligen wurde von der Ärztekammer stattgegeben.

Es wird im Jahre 2005 erstmals zwei Prüfungstermine geben, und zwar:

Samstag, 23. April 2005 und Samstag, 5. November 2005 jeweils um 10 Uhr s. t.

Ort: Ärztekammer für Niederösterreich, 1010 Wien, Wipplingerstraße 4

\section{Prüfungsablauf:}

Geprüft wird mittels Muliple-Choice-Fragen: Innerhalb von 4 Stunden sind 120 Fragen zu beantworten. Die Beantwortung der Fragen erfolgt nach dem Fragentypus A; d. h. es ist aus 5 Wahlmöglichkeiten eine richtige Antwort herauszufinden.

\section{Bewertung:}

Die Bewertung erfolgt ausschließlich mit „bestanden“ oder ,nicht bestanden“. Innerhalb von 8 Wochen ab dem Prüfungstermin werden die Kandidaten vom Prüfungsergebnis schriftlich verständigt. Falls das Gesamtprüfungsergebnis gleich im Anschluss an die Prüfung festgestellt werden kann, ist es möglich, das Ergebnis dem Kandidaten - unabhängig von der schriftlichen Mitteilung - gleich mündlich mitzuteilen. Telefonische Auskünfte sind nicht möglich.

\section{Vorbereitungsmöglichkeiten:}

Grundsätzlich sollte das im Laufe der Ausbildung gesammelte Wissen und die gesammelten Erfahrungen ausreichen, die Facharztprüfung zu bestehen. Die Facharztprüfung dient

\section{Bericht Eye Camp Oshikango, Namibia, Juli 2004}

Abflug Frankfurt - Windhoek am 24. 7. 2004, von Windhoek mit dem Leihwagen $850 \mathrm{~km}$ nach Norden zur Grenzstadt zwischen Namibia und Angola namens Oshikango.

Engela District Hospital, gebaut mit finnischer Unterstützung, mit guter Ausstattung: die beiden Operationssäle wurden für eine Woche für Augenoperationen zur Verfügung gestellt ( $1 \times$ Unterbrechung wegen Sectio): pro Saal 2 gute ZEISS-Operationsmikroskope, Klimaanlage, Fließwasser zur Instrumentensäuberung, 2 Sterilisatoren.

Rekrutierung der PatientInnen durch Ophthalmic Assistents vor einigen Wochen. Unterbringung in Zimmern und Schlafsälen auf Matratzenlagern. Aufklärung der PatientInnen (,Subject") und Einverständniserklärung mittels Fingerabdruckes. Anziehen eines frischen Leintuches, welches am Rücken verknüpft wird, barfuß, Haube.

Biometrie, Spritzen des Lokalanästhetikums ebenso durch Ophthalmic Assistents. Okulopression.

Operationen durchwegs ECCE mit korneoskleralem Tunnelschnitt und Kernexpression. Alle Katarakte entweder matur, hypermatur oder intumeszent, $25 \%$ mit Pseudoexfoliation, tws. Pterygien, tws. droplet climate keratopathy. Zum Teil $(2 / 3)$ beidseits blind, zum Teil $(1 / 2)$ bereits bei Vorgängercamps einseitig operiert worden. Großteils alte bis nicht der Lehrbuchabfrage, sondern soll vor allem jene Kompetenzen überprüfen, die den Facharzt befähigen, auf Grund seiner Ausbildung selbstständig und eigenverantwortlich den alltäglichen Anforderungen gerecht zu werden.

Als Literatur wird ein Lehrbuch der allgemeinen Ophthalmologie empfohlen wie z. B. Kanski - Lehrbuch der klinischen Ophthalmologie, Georg-Thieme-Verlag 1996 oder alternativ Collins Augenheilkunde, Springer-Verlag 2001 bzw. Axenfeld/Pau - Lehrbuch der Augenheilkunde, GustavFischer-Verlag 1992.

Es wird darauf hingewiesen, dass sich rasch entwickelnde Kapitel der Augenheilkunde (refraktive Chirurgie) in diesen Büchern nicht am letzten Stand sein können.

Zum Thema Medizinrecht und Gutachten wird von Univ.Prof. Dr. Radner (s. Mitglieder des Prüfungsausschusses) ein Kurs angeboten werden.

Andere Kurse, wie Kontaktlinsenkurs, Neuroophthalmologischer Kurs, Refraktionskurs, sind sinnvoll, aber nicht Pflicht.

\section{Weitere Informationen:}

Homepage der Akademie der Ärzte: www.arztakademie.at oder www.augen.at.

Es wird empfohlen, zum frühest möglichen Zeitpunkt anzutreten.

Wann darf man antreten?

Der Prüfungskandidat muss zum Zeitpunkt der Prüfungsanmeldung ( 5 Wochen vor der Prüfung) 58 Monate Ausbildung im Haupt- oder Gegenfach vorweisen können.

Univ-Prof. Dr. Michael Radda

Vorsitzender des fachspezifischen Prüfungsausschusses

sehr alte PatientInnen ( $>70$ Jahre), sehr wenig traumatische Katarakte.

Vor den Operationen Visite, tws. mit Spaltlampe. Keine Infektionen.

Operationen von Montag bis Freitag von morgens bis abends, Verpflegung durch die Anstaltsküche. In Summe 261 Katarakt-Operationen.

Teilnehmer: Verantwortlich lokal: Dr. Helena Ndume, Leiterin der Augenklinik Windhoek und des Programmes „Prevention of Blindness"

Dr. Pauline Nyati aus Oshakati (60 km entfernt)

Univ.-Prof. Dr. Anton Haas, Univ.-Augenklinik Graz

DGKS Gabriele Haas

DGKS Rosita Aberer

Dr. Michael Kern, Leoben

Univ.-Prof. Dr. Christoph Faschinger, Univ.-Augenklinik Graz.

Namibia hat 1,7 Millionen Einwohner, 5 Augenärzte (3 Windhoek, 1 Swakopmund, 1 Oshakati). Jährlich finden 4 Eye Camps in den dicht besiedelten Städten des Nordens statt (Rundu oder Katima Mulilo, Grootfontein, Oshakati, Opuwo), tws. mit Hilfe von SEE International (Surgical Eye Expeditions). 